\title{
Riesgo cardiovascular durante el climaterio y la menopausia en mujeres de Santa Cruz del Norte, Cuba
}

\author{
Jonathan Hernández $N^{1}{ }^{1}$, Magel Valdés $Y^{2}$ \\ ${ }^{1}$ Policlínico-Hospital Alberto Fernández Valdés. Santa Cruz Del Norte, Mayabeque, Cuba. ${ }^{2}$ Hospital Dr. Luis Díaz Soto. \\ Habana del Este, La Habana. Cuba.
}

\section{RESUMEN}

Antecedentes: El estudio del climaterio se ha elevado por ser un problema socio-demográfico dado el incremento de la esperanza de vida de la mujer. Objetivo: Identificar factores de riesgo cardiovasculares en mujeres climatéricas y menopáusicas de Santa Cruz del Norte en el período 2011 y 2012. Método: Se realizó un estudio analítico transversal con un universo de 317 mujeres atendidas en consulta y una muestra escogida al azar de 186 divididas en un grupo de 93 pacientes en etapa del climaterio o menopausia y 93 mujeres entre 20 y 39 años. Los datos se recolectaron mediante encuesta y para el análisis estadístico se emplearon medidas descriptivas y pruebas inferenciales. Resultados: Las pacientes climatéricas presentaron significativamente mayor circunferencia de cintura $(t=5,2696 ; p=0,0000)$, índice cintura-cadera $(t=2,2070 ; p=0,0298)$, índice de masa corporal $(t=7,1652 ; p=0,0000)$, y valores de colesterol $(t=4,2531$; $\mathrm{p}=0,0001)$, triglicéridos ( $\mathrm{t}=2,5980 ; \mathrm{p}=0,0109)$ y glicemia $(\mathrm{t}=2,5274 ; \mathrm{p}=0,0132)$. Se observó correlación fuerte y significativa entre la edad y los valores de índice cintura-cadera $(r=0,8116 ; p=0,0000)$ y de glicemia $(r=0,8400 ; p=0,0000)$. Hubo una débil pero significativa correlación entre la edad y la circunferencia de cintura $(r=0,2875 ; p=0,0000)$, índice de masa corporal $(r=0,3246 ; p=0,0000)$ y colesterol $(r=0,2876 ; p=0,0000)$. Hubo más fumadoras entre las climatéricas $(p=0,0360)$ y ambos grupos fueron similares en tensión arterial, hábitos alimentarios y actividad física. Conclusiones: Durante el climaterio y menopausia se produce un incremento en algunos valores antropométricos y metabólicos, que incrementan el riesgo de enfermedades cardiovasculares en esta etapa.

\section{PALABRAS CLAVES: Climaterio, menopausia, factores de riesgo cardiovasculares}

\section{SUMMARY}

Background: The study of the climacteric has risen, being a social and demographic problem due to the increase of the life expectancy of women. Aims: To identify cardiovascular risk factors during climacteric and menopause in women from North's Santa Cruz in 2011 and 2012. Methods: A cross-sectional analytical study was conducted with a universe of 317 women attended in consultation and a random sample of 186 divided in a group of 93 patients in climacteric or menopause stage and other of 93 women between 20 and 39 years old. The data were collected by survey, and for the statistical analysis a descriptive measures and inferential tests were used. Results: Climacteric patients presented significantly greater waist circumference $(t=5.2696 ; p=0.0000)$, waist-hip index $(t=2.2070 ; p=0.0298)$, body mass index $(t=7.1652$; $p=0.0000)$, and values of cholesterol $(t=4.2531 ; p=0.0001)$, triglycerides $(t=2.5980 ; p=0.0109)$ and fasting glucose $(t=2.5274 ; p=0.0132)$. It was observed strong and significant correlation between age and values of 
waist-hip index $(r=0.8116 ; p=0.0000)$ and fasting glucose $(r=0.8400 ; p=0.0000)$. There was a weak but significant correlation between age and waist circumference $(r=0.2875 ; p=0.0000)$, body mass index $(r=0.3246$; $p=0.0000)$ and cholesterol $(r=0.2876 ; p=0.0000)$. There were more smokers between climacteric $(p=0.0360)$ and both groups were similar in blood pressure, nourishing habits and physical activity. Conclusion: During the climacteric and menopause, an increase in some anthropometric and metabolic values take place, that increases the risk of cardiovascular diseases in this stage.

\section{KEY WORDS: Climacteric, menopause, cardiovascular risk factors}

\section{INTRODUCCIÓN}

La esperanza de vida ha ido en aumento en los últimos años y la longevidad trae un mayor número de mujeres que llegan al climaterio y menopausia, como también un incremento en el riesgo de enfermedades cardiovasculares (1).

La tasa de enfermedad cardiovascular como causa de muerte en mujeres estadounidenses aumentó de $30 \%$ en 1997 a $54 \%$ en 2009 , y la tasa de mortalidad ajustada por cardiopatía coronaria en mujeres, fue de 95,7 por 100.000 mujeres en 2007 (2).

El aumento en la enfermedad cardiovascular relacionado con la edad de la mujer se ha vinculado con aumento en las concentraciones de colesterol, incidencia de hipertensión, ganancia de peso e insulino-resistencia. Otros factores involucrados en las enfermedades cardiacas en la etapa el climaterio y menopausia son el tabaquismo, alcoholismo, sedentarismo, sobrepeso, obesidad y estrés $(3,4)$.

Aunque las investigaciones epidemiológicas han examinado si los niveles de factores de riesgo aumentan significativamente a medida que las mujeres cambian del estado premenopáusico al posmenopáusico, muchas no han sido concluyentes, por lo que realizamos este estudio con el objetivo de determinar los factores de riesgo cardiovasculares en mujeres cubanas climatéricas y menopáusicas pertenecientes al municipio de Santa Cruz del Norte en el período 2011 y 2012.

\section{PACIENTES Y MÉTODO}

Se realizó un estudio descriptivo de corte transversal a partir de un universo de 317 mujeres atendidas en consulta de ginecología del municipio Santa Cruz del Norte, en el período comprendido entre el $1^{\circ}$ de enero de 2011 al 31 de diciembre de 2012. Se escogió una muestra de 186 pacientes a través de un muestreo probabilístico aleatorio simple que se dividió en dos grupos: Grupo A formado por 93 pacientes en etapa del climaterio o menopausia (rango: 45 y 59 años) y Grupo B constituido por 93 pacientes en edad fértil (rango: 20 y 39 años).

Los datos se recolectaron a través de un for- mulario que fue llenado mediante encuesta a las pacientes y la realización de exámene físico y complementarios. Se estudiaron: A. Variables antropométricas, como circunferencia de cintura, índice cintura-cadera e índice de masa corporal. B. Variables metabólicas, como colesterol, triglicéridos y glicemia de ayuno. C. Otros, como hábitos alimentarios, actividad física, hábito de fumar y tensión arterial.

Los hábitos alimentarios se consideraron de la siguiente manera: Buenos (ingestión de abundantes frutas y vegetales diariamente), Regulares (ingestión de más carbohidratos que vegetales y frutas pero con poca grasa) y Malos (ingestión de alimentos ricos en grasa diariamente y ocasionalmente vegetales y frutas).

La actividad física se consideró de la siguiente manera: Siempre (ejercicios físicos diarios), Frecuentemente (2 a 3 veces por semana), A veces (2 a 3 veces en el mes) y Nunca (no realiza ejercicios físicos).

Los datos se procesaron empleando el paquete estadístico SPSS versión 15.0 y se empleó la media aritmética y desviación estándar para resumir las variables cuantitativas y el porcentaje para las cualitativas. Para el análisis estadístico inferencial se empleo el test de Student como prueba de hipótesis sobre diferencias de medias con desviación estándar poblacional desconocida para las variables cuantitativas y la prueba de homogeneidad mediante el estadígrafo chi para las variables cualitativas; todo ello con un nivel de confiabilidad del $95 \%$.

Para establecer la relación entre la edad y las demás variables cuantitativas se empleó el coeficiente de correlación lineal muestral y la prueba de hipótesis para el coeficiente de correlación y de la regresión igualmente a través del test de Student con un nivel de confiabilidad del $95 \%$.

Para realizar el estudio se solicitó la aprobación del comité de ética y del consejo científico de nuestra institución, así como el consentimiento de todas las pacientes involucradas en el mismo, explicando de manera clara su importancia y se garantizó la discreción en el manejo de la información; además se siguieron todos los principios de la ética médica profesional. 


\section{RESULTADOS}

Se observó que la media y desviación estándar de las medidas antropométricas fueron significativamente mayores $(p<0,05)$ en las pacientes climatéricas o menopáusicas que en las premenopáusicas; sobre todo el índice de masa corporal (Grupo A $31,2 \pm 2,8 \mathrm{Kg} / \mathrm{m} 2$ vs. Grupo B 25,3 $\pm 5,8 \mathrm{Kg} / \mathrm{m} 2$ ) y la circunferencia de cintura (Grupo A 92,9 $\pm 9,3 \mathrm{~cm}$ vs. Grupo B 87,4 $\pm 8,7 \mathrm{~cm}$ ) (Tabla I).

Los valores de colesterol, triglicéridos y glicemia en ayunas fueron significativamente mayores en las pacientes climatéricas o menopáusicas que en las premenopáusicas. No hubo diferencias estadísticamente significativas desde el entre ambos grupos en las cifras de tensión arterial sistólica ni diastólica (Tabla II).
La correlación entre la edad de las pacientes y las variables antes mencionadas evidenció una fuerte relación directamente proporcional entre la edad y el índice cintura-cadera $(r=0,8116$; $p=0,0000)$ e igualmente con el valor de glicemia $(r=0,8400 ; p=0,0000)$; la circunferencia de cintura, índice de masa corporal y valores de colesterol solo mostraron una relación débil directamente proporcional con el incremento de la edad, no hubo correlación con los valores de triglicéridos y de tensión arterial con la edad (Tabla III).

Hubo una diferencia significativa entre ambos grupos con respecto al hábito de fumar, con 63 pacientes para un $67,7 \%$ en el grupo A contra 49 pacientes para un $52,7 \%$ en el grupo $B(p=0,0360)$; no hubo diferencias entre ambos grupos en otras variables relacionadas con el estilo de vida, tales como hábitos alimentarios y actividad física (Tabla IV).

Tabla I

DISTRIBUCIÓN DE LAS MUJERES SEGÚN MEDIDAS ANTROPOMÉTRICAS

\begin{tabular}{|c|c|c|c|}
\hline \multirow{2}{*}{$\begin{array}{l}\text { Medidas } \\
\text { antropométricas }\end{array}$} & \multicolumn{2}{|c|}{ Media $( \pm D E)$} & \multirow[b]{2}{*}{$t($ valor $p)$} \\
\hline & Grupo A & Grupo B & \\
\hline Circunferencia de cintura (cm) & $92,9(9,3)$ & $87,4(8,7)$ & $5,2696(0,0000)$ \\
\hline Índice cintura-cadera & $0,91(0,17)$ & $0,86(0,07)$ & $2,2070(0,0298)$ \\
\hline Índice de masa corporal (Kg/m2) & $31,2(2,8)$ & $25,3(5,8)$ & $7,1652(0,0000)$ \\
\hline
\end{tabular}

Tabla II

DISTRIBUCIÓN DE LAS MUJERES SEGÚN TENSIÓN ARTERIAL Y PARÁMETROS HEMOQUÍMICOS

\begin{tabular}{|c|c|c|c|}
\hline \multirow{2}{*}{$\begin{array}{l}\text { Tensión arterial y } \\
\text { complementarios }\end{array}$} & \multicolumn{2}{|c|}{ Media $( \pm D E)$} & \multirow[b]{2}{*}{$\mathrm{t}$ (valor p) } \\
\hline & Grupo A & Grupo B & \\
\hline Tensión arterial sistólica (mmHg) & $124(12)$ & $123(12)$ & $1,9029(0,0602)$ \\
\hline Tensión arterial diastólica $(\mathrm{mmHg})$ & $81(7)$ & $80(7)$ & $1,6830(0,0958)$ \\
\hline Colesterol (mmol/L) & $5,9(0,6)$ & $5,6(0,4)$ & $4,2531(0,0001)$ \\
\hline Triglicéridos (mmol/L) & $1,6(0,5)$ & $1,5(0,4)$ & $2,5980(0,0109)$ \\
\hline Glicemia (mmol/L) & $5,4(2,0)$ & $5,0(1,2)$ & $2,5274(0,0132)$ \\
\hline
\end{tabular}


Tabla III

CORRELACIÓN LINEAL ENTRE EDAD DE LA MUJER Y ANTROPOMETRÍA, TENSIÓN ARTERIAL Y PARÁMETROS HEMOQUÍMICOS

\begin{tabular}{lc}
\hline Factores & $r$ (valor p) \\
\hline Circunferencia de cintura $(\mathrm{cm})$ & $0,2875(0,0000)$ \\
Índice cintura-cadera & $0,8116(0,0000)$ \\
Índice de masa corporal $(\mathrm{Kg} / \mathrm{m} 2)$ & $0,3246(0,0000)$ \\
Tensión arterial sistólica $(\mathrm{mmHg})$ & $0,0770(0,2959)$ \\
Tensión arterial diastólica $(\mathrm{mmHg})$ & $0,0666(0,3664)$ \\
Colesterol $(\mathrm{mmol} / \mathrm{L})$ & $0,2876(0,0000)$ \\
Triglicéridos $(\mathrm{mmol} / \mathrm{L})$ & $0,1101(0,1346)$ \\
Glicemia $(\mathrm{mmol} / \mathrm{L})$ & $0,8400(0,0000)$ \\
\hline
\end{tabular}

$\mathrm{p}=0,0132$ ), como también el hábito tabáquico $(p=0,0360)$. En general estos resultados son concordantes con la literatura y que analizaremos a continuación.

Allison y cols (5), mostraron que la circunferencia de cintura es un marcador sustituto de obesidad abdominal por su correlación con la grasa abdominal y su asociación con el riesgo cardiometabólico; consideran que esta medida por encima de $88 \mathrm{~cm}$ se asocia a factores de riesgo aterogénicos como dislipidemia, hipertensión arterial e insulinorresistencia y que la misma se incrementa con la edad, con más frecuencia en la mujer posmenopáusica.

Feng y cols (6), encontraron un porcentaje mayor de grasa abdominal en las pacientes posmenopáusicas al observar un índice cintura-cadera por enci-

Tabla IV

DISTRIBUCIÓN DE LAS MUJERES SEGÚN ESTILO DE VIDA

\begin{tabular}{lccr}
\hline Estilo de Vida & $\begin{array}{c}\text { Grupo A } \\
\mathrm{n}(\%)\end{array}$ & $\begin{array}{c}\text { Grupo B } \\
\mathrm{n}(\%)\end{array}$ & Valor p \\
\hline Hábitos alimentarios & $\mathrm{X}^{2}=2,89$ & & $\mathrm{p}=0,2357$ \\
- Buenos & $13(14,0)$ & $21(22,6)$ & 0,1291 \\
- Regulares & $69(74,2)$ & $65(69,9)$ & 0,5134 \\
- Malos & $11(11,8)$ & $7(7,5)$ & 0,3212 \\
Actividad física & $\mathrm{X}^{2}=3,60$ & $\mathrm{p}=0,3075$ \\
- Siempre & $5(5,4)$ & $9(9,7)$ & 0,2663 \\
- Frecuentemente & $6(6,5)$ & $9(9,7)$ & 0,4192 \\
- A veces & $17(18,3)$ & $22(23,7)$ & 0,3678 \\
- Nunca & $65(69,9)$ & $53(57,0)$ & 0,0677 \\
Hábito de fumar & $\mathrm{X}^{2}=4,40$ & & $\mathrm{p}=0,0360$ \\
- Sí & $63(67,7)$ & $49(52,7)$ & 0,0360 \\
- No & $30(32,3)$ & $44(47,3)$ &
\end{tabular}

\section{DISCUSIÓN}

Los resultados de nuestro estudio muestran que hay diferencias significativas en algunos factores de riesgo cardiovascular entre mujeres climatéricas y en menopausia comparadas con mujeres menores de 40 años. Destacando los factores de riesgo antropométricos como circunferencia de cintura ( $t=5,2696 ; p=0,0000)$, índice cintura-cadera $(t=2,2070 ; \quad p=0,0298)$ e índice de masa corporal $(t=7,1652 ; p=0,0000)$; los factores de riesgo metabólicos como colesterol ( $t=4,2531 ; p=0,0001)$, triglicéridos $(t=2,5980 ; p=0,0109)$ y glicemia $(t=2,5274$; ma de $0,85 \mathrm{~cm}$ en el $71,4 \%$ de los casos, contra un $34,8 \%$ en las premenopáusicas. Igualmente, Castañeda y Amigo (7), observaron como uno de los factores de riesgo vascular más importantes, el índice cintura-cadera mayor de $0,85 \mathrm{~cm}$ en el $34 \%$ de las pacientes, sobre todo en las posmenopáusicas.

Karim y cols (8), encontraron un índice de masa corporal de $28,8 \pm 5,4 \mathrm{Kg} / \mathrm{m} 2$ en las pacientes posmenopáusicas significativamente mayor que en las premenopáusicas. Por otro lado, Ramos y cols (9), evidenciaron la obesidad abdominal en el $48,0 \% \pm$ $3,36 \%$ (IC: 44,3 a $51,7 \%$ ) de las mujeres de su estudio, sobre todo en las posmenopáusicas. 
En un reciente estudio realizado en Irán por Sarrafzadegan y cols (10), se encontró que las mujeres premenopáusicas tienen significativamente menor índice de masa corporal, circunferencia de cintura e índice cintura-cadera que las posmenopáusicas. En este estudio se puso de manifiesto que en las mujeres posmenopáusicas los factores bioquímicos, como glicemia en ayunas y perfil lipídico, son significativamente mayores que en las premenopáusicas.

Un estudio realizado por Dasgupta y cols (11), concluyó que la transición hacia la menopausia trae consigo anomalías en la composición corporal total caracterizada por un incremento de la masa adiposa corporal y adiposidad central dado por un aumento significativo en el índice de masa corporal, circunferencia de cintura y porcentaje de grasa; esto crea una atmósfera compatible para un metabolismo anormal y agravamiento de los factores de riesgo cardio-metabólicos. Así, concluyeron que el estado menopáusico y la obesidad asociada es el principal predictor de alteraciones metabólicas de riesgo cardiovascular en las mujeres menopáusicas.

Del Valle (12), presentó como la glicemia y la diabetes mellitus tipo 2 se incrementa con la edad en ambos sexos, siendo predominante el sexo femenino sobre el masculino. Hoffmann \& Icks (13) plantean un predominio del sexo femenino en respuesta a una mayor presencia de obesidad con aumento de la resistencia a la insulina, mala distribución de la grasa corporal, pérdida de la función protectora de los estrógenos y mayor expectativa de vida.

Con respecto al perfil lipídico en mujeres posmenopáusicas, Barrios y cols (14), observaron altos porcentajes de colesterol total (CT) en el 83,6\%, HDL-colesterol en 54,1\%, LDL-colesterol en 54,1\% y triglicéridos en 37,7\%; igualmente encontraron los índices aterogénicos LDL/HDL elevados en el $75,4 \%$ y CT/HDL en $67,2 \%$ de los casos de su estudio. De esa manera, concluyeron que las mujeres posmenopáusicas evaluadas presentaron un perfil lipídico aterogénico en situación de riesgo para enfermedades cardiovasculares.

Kunstmann y cols (15), mostraron que después de la quinta década de la vida, las mujeres tienen mayores niveles plasmáticos de colesterol total y menores de colesterol HDL asociado a la menopausia. Por otra parte, la hipertrigliceridemia es un potente factor de riesgo independiente en la mujer a esta edad.

Según Padró Suárez y Pelegrín Martínez (16), el cambio en el perfil lipídico que se observa en la posmenopausia se caracteriza por el aumento de triglicéridos, del colesterol unido a lipoproteínas de baja densidad (LDL) y de la lipoproteína $A$, y por el descenso del colesterol unido a lipoproteínas de alta densidad (HDL). Esta modificación del perfil lipídico se relaciona con mayor riesgo de enfermedad cardiovascular.
Para Ballesteros Hernández y Guirado Blanco (17), la hipoestrogenemia no solo favorece el incremento de las LDL, sino también su oxidación, por lo cual la aterogénesis está favorecida por dos mecanismos fundamentales: uno es la oxidación de las LDL, cuya captación por los macrófagos aumenta cuando están oxidadas, y segundo, por las alteraciones del metabolismo de la insulina que ocurren en la posmenopausia por la disminución de los estrógenos.

May y cols (18), evaluaron los valores del índice remanente de apolipoproteína $\mathrm{A} 1$ como un nuevo marcador de riesgo cardiovascular en la mujer climatérica y observaron que este fue un predictor significativo de muerte por infarto del miocardio en mujeres mayores de 50 años a corto y mediano plazo.

Lejsková y cols (19), observaron un incremento en los parámetros antropométricos e índices de lípidos plasmáticos en las mujeres posmenopáusicas, estrechamente relacionados con el incremento de la edad; para estos autores, la menopausia puede plantear un riesgo a las mujeres a través de un agrupamiento de factores de riesgo cardiovasculares más allá del simple envejecimiento.

Sowers y cols (20), observaron una relación directamente proporcional entre el índice cintura-cadera y la edad, considerando que este se encuentra mucho más elevado en las pacientes posmenopáusicas que en las premenopáusicas.

Cagnacci y cols (21), evidenciaron que los niveles de glicemia fueron significativamente superiores en las pacientes posmenopáusicas al compararlas con las premenopáusicas, incluso observaron que a medida que se avanzaba en la edad de la menopausia, mayores eran los niveles de glicemia, por lo que consideran que la elevación de los niveles de glucosa en sangre es uno de los factores de riesgo de ateroesclerosis que se presentan con mayor frecuencia en las pacientes posmenopáusicas.

Desde hace años se viene señalando que el déficit estrogénico es el principal causante de la mayor susceptibilidad femenina en la época posmenopáusica para padecer enfermedades cardiovasculares; así, los estrógenos potencian la acción de las prostaciclinas, incrementan la producción de óxido nítrico, inhiben la enzima de conversión de angiotensina en las células mioepiteliales, disminuyen la reactividad vascular, aumentan la sensibilidad a la insulina e interactúan con el metabolismo lipídico. Además, tienen un papel tanto en la regulación del tono vascular, en el crecimiento de los miocitos vasculares, así como en el incremento de la sensibilidad a la sal. Todos estos mecanismos podrían explicar el incremento del riesgo cardiovascular en la mujer durante el climaterio y la menopausia $(17,22)$.

El impacto del tabaco en la enfermedad ateroesclerótica es mayor en la mujer que en el hombre; no solo predispone a la ateromatosis sino también 
a los cuadros trombóticos vasculares. Así, Ramos y cols (9), encontraron que el tabaquismo estuvo presente en el 39,5\% $\pm 3,59 \%$ (IC: $35,9 \%$ a $43,1 \%$ ) de las pacientes, sobre todo en las posmenopáusicas. Según Lahoz y otros (23), el tabaco favorece el desarrollo de la aterosclerosis a través de la lesión del endotelio por el monóxido de carbono circulante, el aumento del fibrinógeno y del factor VII, el aumento de la adherencia y la agregabilidad de las plaquetas, el aumento de la oxidación de las lipoproteínas de baja densidad (LDL), el descenso de la concentración de colesterol de las lipoproteínas de alta densidad (HDL) y el efecto vasoconstrictor.

Para Grundy y Deuke (24), la dieta inadecuada puede considerarse como el principal factor exógeno o ambiental que interviene en la etiopatogenia de diversas alteraciones del metabolismo lipídico relacionadas con un riesgo aumentado de enfermedad aterosclerótica; también puede intervenir en el desarrollo de la aterosclerosis a través de su influencia sobre otros factores de riesgo (obesidad, diabetes, hipertensión arterial), sobre el sistema de la coagulación y la susceptibilidad oxidativa de las LDL.

Colpani y cols (25), observaron que la inactividad física se asoció a un mayor riesgo de sobrepeso y obesidad (OR:2,1; IC95\%:1,233-3,622; $\mathrm{p}=0,006)$ y una circunferencia de cintura mayor de $88 \mathrm{~cm}$ (OR:1,7; IC95\%:1,054-2,942; $p=0,03$ ) en las mujeres perimenopáusicas; igualmente observaron un incremento del riesgo de diabetes mellitus (OR:2,7; IC95\%:1,233-6,295; p=0,014) y síndrome metabólico (OR:2,5; IC95\%:1,443-4,294; $p=0,001$ ) en estas pacientes. Estos autores concluyeron que la actividad física habitual promueve una disminución del riesgo de enfermedades cardiovasculares y diabetes en las mujeres de edad mediana.

Un estudio realizado por Miyaki y otros (26), demostró que el ejercicio aeróbico habitual conlleva a un incremento en los niveles plasmáticos de la proteína de acción antinflamatoria pentraxin 3 , mejorando secundariamente, por la acción de esta proteína, los niveles de HDL-colesterol, disponibilidad máxima de oxígeno y distensibilidad arterial en las mujeres posmenopáusicas.

\section{CONCLUSIÓN}

Durante el climaterio y menopausia se produce un incremento en algunos valores antropométricos y metabólicos, que incrementan el riesgo de enfermedades cardiovasculares en mujeres cubanas, concordante con lo descrito en la literatura internacional sobre el tema.

\section{REFERENCIAS}

1. Lugones Botell M. La salud de la mujer en el climaterio y la menopausia. La Habana: Editorial Científico Técnica; 2006.
2. Lloyd Jones D, Adams R, Carnethon M, De Simone G, Ferguson TB, Flegal K, et al. Heart disease and stroke statistics-2009 update: a report from the American. Heart Association Statistics Committee and Stroke Statistics Subcommittee. Circulation 2009;119:e21e181.

3. Mosca L, Mochari-Greenberger H, Dolor RJ, Newby LK, Robb KJ. Twelve-year follow-up of American women's awareness of cardiovascular disease risk and barriers to heart health. Circ Cardiovasc Qual Outcomes 2010;3:120-7.

4. Matthews KA, Crawford SL, Chae CU, Everson-Rose SA, Sowers MF, Sternfeld B, et al. Are changes in cardiovascular disease risk factors in midlife women due to chronological aging or to the menopausal transition? Results from the Study of Women's Health Across the Nation (SWAN). J Am Coll Cardiol 2009;54:2366-73.

5. Allison $\mathrm{H}$, Christian D, Mochari $\mathrm{H}$, Mosca JL. waist circumference, body mass index, and their association with cardiometabolic and global risk. J Cardiometabolic Synd 2009;4:12-4.

6. Feng $\mathrm{Y}$, Hong $\mathrm{X}$, Wilker $\mathrm{E}$, Li Z, Zhang W, Jin D, et al. Effects of age at menarche, reproductive years, and menopause on metabolic risk factors for cardiovascular diseases. Atherosclerosis 2008;196:590-7.

7. Castañeda Gueimonde C, Amigo González R. Calidad de vida y mujer de edad mediana. Rev Avan Científ [en línea] 2006. [citada 2013 May 12] 9(9): [11 pantallas]. Disponible en: http://www.atenas.inf.cu/Publicaciones/ avanzada/Num2_2006/Articulos/ramal.htm.

8. Karim R, Hodis HN, Stanczyk FZ, Lobo RA, Mack WJ. Relationship between serum levels of sex hormones and progression of subclinical atherosclerosis in postmenopausal women. J Clin Endocrinol Metab 2008;93:131-8.

9. Ramos Morales LE, Gallardo Pérez UJ, Cabrera Zamora J, Salgado Boris L, Adam Simón D, Mahía Vilas $M$, et al. Riesgo aterogénico y enfermedad cardiocerebrovascular y arterial periférica en mujeres de edad mediana. Rev Cubana Angiol Cir Vasc [en línea] 2011. [citada 2013 May 12] 12(1): [9 pantallas]. Disponible en: http://bvs.sld.cu/revistas/ang/vol12_01_11/ ang03111.htm.

10. Sarrafzadegan N, Khosravi-Boroujeni H, Esmaillzadeh A, Sadeghi M, Rafieian-Kopaei M, Asgary S. The association between hypertriglyceridemic waist phenotype, menopause, and cardiovascular risk factors. Arch Iran Med 2013;16:161-6.

11. Dasgupta S, Salman M, Lokesh S, Xaviour D, Saheb SY, Prasad BV, et al. Menopause versus aging: The predictor of obesity and metabolic aberrations among menopausal women of Karnataka, South India. J Midlife Health 2012;3:24-30.

12. Del Valle M. Epidemiología de la diabetes. En: XIX Congreso Latinoamericano de Patología Clínica/ML. La Habana: ALAPAC; 2009.

13. Hoffmann F, Icks A. Diabetes 'epidemic' in Germany? A critical look at health insurance data sources. Exp Clin Endocrinol Diabetes 2012;120:410-5.

14. Barrios Y, Meertens L, Salim de Moron A, Díaz N. Proteína $C$ reactiva, su relación con el estado nutricional y perfil lipídico en mujeres posmenopáusicas. Rev Venez Endocrinol Metab 2011;9:106-11.

15. Kunstmann S, de Grazia R, Gainza D. Aterosclerosis en la mujer: factores de riesgo y prevención. Rev Chil Cardiol 2012;31:142-7. 
16. Padró Suárez L, Pelegrín Martínez AA. Perimenopausia y riesgo cardiovascular. Medisan 2011;15(10):1485-92.

17. Ballesteros Hernández M, Guirado Blanco O. Los estrógenos como protectores cardiovasculares. Medicentro Electrón [en línea] 2012. [citada 2013 May 12] 16(3): [6 pantallas]. Disponible en: http://medicentro.vcl.sld.cu/paginas\%20de\%20acceso/Sumario/ ano\%202012/v16n3a12/estrogenos.htm.

18. May HT, Nelson JR, Kulkarni KR, Anderson JL, Horne $\mathrm{BD}$, Bair TL, et al. A new ratio for better predicting future death/myocardial infarction than standard lipid measurements in women $>50$ years undergoing coronary angiography: the apolipoprotein $\mathrm{A} 1$ remnant ratio (Apo A1/ [VLDL3+IDL]). Lipids Health Dis 2013; 2: 5-67.

19. Lejsková M, Alušík S, Valenta Z, Adámková S, Pitha J. Natural postmenopause is associated with an increase in combined cardiovascular risk factors. Physiol Res 2012;61:587-96.

20. Sowers MF, Zheng H, Tomey K, Symons J. Changes in body composition in women over six years at midlife: ovarian and chronological aging. J Clin Endocrinol Metabol 2007;92:894-901.
21. Cagnacci A, Cannoletta M, Palma F, Zanin R, Xholli A, Volpe A. Menopausal symptoms and risk factors for cardiovascular disease in postmenopause. Climacteric 2012;15:157-62.

22. Pappa T, Alevizaki M. Endogenous sex steroids and cardio- and cerebro-vascular disease in the postmenopausal period. Eur J Endocrinol 2012;167:145-56.

23. Lahoz C, Mostaza JM. Enfermedad arterial no coronaria (I): La aterosclerosis como enfermedad sistémica. Rev Esp Cardiol 2007; 60:184-95.

24. Grundy SM, Deuke MA. Dietary influences on serum lipids and lipoproteins. J Lipid Res 2010;51:1213-9.

25. Colpani V, Oppermann K, Spritzer PM. Association between habitual physical activity and lower cardiovascular risk in premenopausal, perimenopausal, and postmenopausal women: a population-based study. Menopause 2013;20:525-31.

26. Miyaki A, Maeda S, Choi Y, Akazawa N, Tanabe $Y$, Ajisaka R. Habitual aerobic exercise increases plasma pentraxin 3 levels in middle-aged and elderly women. Appl Physiol Nutr Metab 2012;37:907-11. 